\title{
Efeito protetor da acetamida em bovinos indica monofluoroacetato como princípio tóxico de Palicourea marcgravii (Rubiaceae) ${ }^{1}$
}

\author{
Tiago C. Peixoto ${ }^{2 *}$, Vivian A. Nogueira ${ }^{3}$, Saulo A. Caldas ${ }^{4}$, Ticiana N. França ${ }^{3}$, \\ Bruno L. Anjos ${ }^{5}$, Ana Paula Aragão ${ }^{6}$ e Paulo V. Peixoto ${ }^{7}$
}

\begin{abstract}
Peixoto T.C., Nogueira V.A., Caldas S.A., França T.N., Anjos B.L., Aragão A.P. \& Peixoto P.V. 2012. [Protective effect of acetamide in cattle indicates that monofluoroacetate is the toxic principle of Palicourea marcgravii (Rubiaceae).] Efeito protetor da acetamida em bovinos indica monofluoroacetato como princípio tóxico de Palicourea marcgravii $(\mathrm{Ru}-$ biaceae). Pesquisa Veterinária Brasileira 32(4):219-328. Projeto Sanidade Animal Embrapa/ UFRRJ, Seropédica, RJ 23890-000, Brazil. E-mail: tiagocpeixoto@yahoo.com.br

The aim of the study was to evaluate and compare the protective effect of acetamide in experimental poisoning by fresh leaves of Palicourea marcgravii and monofluoroacetate (MF) in catlle, in order to prove in a practical way that this compound is the toxic principle responsible for the clinical signs and death of animals that ingested the plant. MF was administered orally in single doses of $0.5 \mathrm{mg} / \mathrm{kg}$ to three cows; a few minutes later, two of these cows received, orally, single doses of acetamide $(0.38$ or $2.0 \mathrm{~g} / \mathrm{kg})$. Two other cows ingested $1.0 \mathrm{~g} / \mathrm{kg}$ of $P$. marcgravii, and one of these cows received some minutes later $1.0 \mathrm{~g} /$ $\mathrm{kg}$ of acetamide. Adequate doses of acetamide, administered right after the poisoning by $P$. marcgravii or MF, were able to prevented the onset of clinical signs and avoid the death of all animals. One week later, the experiments were repeated, but without the antidote. All animals not treated with acetamide showed symptoms of poisoning and died suddenly. MF and P. marcgravii caused the same clinical and pathological picture of "sudden death" in cattle. Clinically, the cattle presented palpitation, abdominal breathing, muscle tremors, engorged jugular vein with positive pulse, pollakiuria, slight loss of balance with sometimes swaying gait, the animals laying down and with the head on their flank. In the "dramatic phase", all the animals fell into lateral decubitus, stretched the limbs, made paddling movements, presented opistotonus, arrhythmia, nystagmus, and died. The "dramatic phase" lasted from 2 to 26 minutes. At postmortem examination, the heart auricles, jugulars and pulmonary veins were slightly to moderately ingurgitated; slight to marked edema of the subserosa was seen in fixation sites of gall bladder to the liver. In one cow, pulmonary edema was observed. Histopathology revealed in all cows slight to marked hydropic-vacuolar degeneration of the epithelial cells of the distal convoluted uriniferous tubules associated with nuclear pyknosis. Coagulation necrosis of individual or groups of hepatocytes and slight to moderate hepatic congestion with numerous shock corpuscles were also observed. The experimental results showed in practice that MF is the toxic principle responsible for the clinical-pathological picture and death of the cattle that ingested P. marcgravii, since acetamide acts as an efficient antidote (antagonistic effect), identical in both poisonings.
\end{abstract}

INDEX TERMS: Monofluoroacetate, acetamide, Palicourea marcgravii, cattle.

\footnotetext{
${ }^{1}$ Recebido em 4 de novembro de 2011.

Aceito para publicação em 8 de dezembro de 2011.

Parte da Tese de Doutorado do primeiro autor, defendida na Universidade Federal Rural do Rio de Janeiro (UFRRJ), Seropédica, RJ Brasil.

${ }^{2}$ Curso de Pós-Graduação em Ciências Veterinárias, Instituto de Veterinária, UFRRJ, Seropédica, RJ 23890-000. Autor para correspondência: tiagocpeixoto@yahoo.com.br

${ }^{3}$ Departamento de Epidemiologia e Saúde Pública, Instituto de Veterinária, UFRRJ, Seropédica, RJ.
}

\footnotetext{
${ }^{4}$ Bolsista de Pós-Doutorado Júnior CNPq, Convênio UFRRJ, Seropédica, RJ.

${ }^{5}$ Laboratório de Patologia Veterinária, Universidade Federal do Pampa (Unipampa) - Campus Uruguaiana, BR 472, Km 592, 97500-970, Uruguaiana, RS, Brasil.

${ }^{6}$ Curso de Pós-Graduação em Ciências Veterinárias, UFRRJ, Seropédica, RJ.

${ }^{7}$ Departamento de Nutrição Animal e Pastagem, Instituto de Zootecnia, Universidade Federal Rural do Rio de Janeiro (UFRRJ), Seropédica, RJ 23890-000.
} 
RESUMO.- 0 presente trabalho teve como objetivo avaliar e comparar o efeito protetor da acetamida nas intoxicações experimentais por monofluoroacetato (MF) e por folhas frescas de Palicourea marcgravii em bovinos, no intuito de confirmar, de forma prática, que esse composto é o princípio tóxico responsável pelo quadro clínico-patológico e pela morte dos animais intoxicados por essa planta. Três bovinos receberam MF, por via oral, na dose de $0,5 \mathrm{mg} / \mathrm{kg}$ e, em seguida, a dois desses animais administraram-se acetamida, por via oral, nas doses de 0,38 e $2,0 \mathrm{~g} / \mathrm{kg}$. Outros dois bovinos receberam $1,0 \mathrm{~g} / \mathrm{kg}$ de $P$. marcgravii, em seguida, a um deles administrou-se $1,0 \mathrm{~g} / \mathrm{kg}$ de acetamida. Acetamida, quando administrada em quantidades suficientes (maior dose), evitou o aparecimento dos sinais clínicos e a morte de todos os animais que receberam MF ou P. marcgravii. Tal efeito protetor foi, de fato, confirmado após uma semana, quando o mesmo protocolo experimental foi repetido, para cada bovino, porém sem a administração de acetamida. Todos os bovinos não tratados com acetamida manifestaram sinais clínicos e morreram subitamente. 0 quadro clínico-patológico manifestado pelos bovinos intoxicados por MF ou P. marcgravii foi semelhante e, caracterizou-se por "morte súbita". Os animais em geral, apresentaram taquicardia, taquipnéia, tremores musculares, jugular repleta com pulso venoso positivo, polaquiúria, instabilidade, perda de equilíbrio, por vezes, cambaleavam e apoiavam a cabeça no flanco. Na fase final, todos os animais deitavam-se e levantavam-se com maior frequencia, deitavam ou caíam em decúbito lateral, esticavam os membros, faziam movimentos de pedalagem, apresentavam respiração ofegante, arritmia, opistótono, nistagmo, mugiam e morriam. A duração da "fase dramática" variou de 2 a $26 \mathrm{~min}$. À necropsia verificaram-se, em geral, aurículas, jugulares, ázigos e pulmonares leve a moderadamente ingurgitadas, leve a acentuado edema da subserosa da vesícula biliar, sobretudo, na sua inserção no fígado, bem como moderada quantidade de líquido espumoso róseo na traquéia e brônquios. 0 exame histopatológico revelou, no rim de todos os animais, leve até acentuada degeneração hidrópico-vacuolar das células epiteliais dos túbulos uriníferos contornados distais associada à picnose nuclear; no fígado, havia leve a moderada congestão, discreta a moderada tumefação e moderada vacuolização de hepatócitos, predominantemente, centrolobular, necrose de coagulação individual ou de grupos de hepatócitos e corpúsculos de choque. Os dados obtidos neste trabalho comprovam, de forma prática, que MF é o princípio tóxico de $P$. marcgravii responsável pelo quadro clínico-patológico e a morte dos animais que ingerem e se intoxicam naturalmente por essa planta, uma vez que a acetamida atua como antídoto eficaz (efeito antagônico) de forma idêntica em ambas as intoxicações.

TERMOS DE INDEXAÇÃO: Monofluoroacetato, acetamida, Palicourea marcgravii, bovinos.

\section{INTRODUÇÃO}

No Brasil são conhecidas, até o momento, 12 plantas que causam "morte súbita" (PBCMS). Nesse grupo, estão incluídas as plantas tóxicas de interesse pecuário mais impor- tantes do país, e que são responsáveis por centenas de milhares de mortes de bovinos, todos os anos. Dentre essas, destaca-se Palicourea marcgravii, por sua elevada toxidez, ampla distribuição, boa palatabilidade e efeito acumulativo (Tokarnia et al. 2000). Desde a década de 1930, P. marcgravii tem sido objeto de diversos estudos, sobretudo, no que se refere à identificação e à quantificação de possíveis substâncias tóxicas ou com ação farmacológica, contudo, ainda hoje, há dúvidas sobre qual substância, dentre as diversas já isoladas, seria, de fato, o princípio tóxico da planta capaz de determinar o quadro clínico-patológico e a morte dos animais intoxicados.

Em 1959, foi detectada no rim de bovinos intoxicados por P. marcgravii, uma lesão designada degeneração hidrópico-vacuolar (DHV) dos túbulos contornados distais (TCD), que foi considerada típica para essa intoxicação, em função de sua distribuição seletiva, quase exclusiva, a esses túbulos e pela marcada picnose nuclear (Döbereiner \& Tokarnia 1959). Mais tarde, verificou-se que tal lesão também aparecia no rim de parte dos animais intoxicados por todas as outras 11 PBCMS. Recentemente, demonstrou-se que o quadro clínico-patológico manifestado por bovinos (Nogueira et al. 2010), ovinos (Peixoto et al. 2010), coelhos (Peixoto, comunicação pessoal) e ratos (Peixoto et al. 2011b) intoxicados experimentalmente com monofluoroacetato (MF) corresponde, em diversos aspectos, ao observado em casos de intoxicação por PBCMS e, que, a típica DHV dos TCD associada à picnose nuclear é uma lesão característica da intoxicação por MF em algumas espécies animais (Nogueira et al. 2011, Peixoto et al. 2011a).

MF, uma das substâncias mais tóxicas já descobertas, tem sido isolado de diversas plantas cuja ingestão determina "morte súbita" em bovinos na África do Sul e na Austrália; no Brasil, essa substância foi identificada por cromatografia em camada delgada em P. marcgravii (Oliveira 1963) e, através de espectroscopia por ressonância magnética nuclear flúor ${ }^{19}\left(\mathrm{RMN}^{19} \mathrm{~F}\right)$ tanto em $P$. marcgravii, quanto em Arrabidaea bilabiata (Krebs et al. 1994) por técnicas cromatográficas; há ainda indícios da presença de MF em Amorimia (Mascagnia) rigida (Cunha 2008). Alguns autores, contudo, acreditam que esse composto não seria o princípio tóxico determinante das mortes dos animais que ingerem essas plantas (Habermehl 1986, González et al. 2000) ou que haveria outras substâncias que poderiam causar a morte dos animais (Peckolt 1868, Corrêa 1869, Guimarães 1934, Hoehne 1939, Mello \& Fernandes 1940, Górniak 1986) ou contribuir para a toxicidade dessas plantas (Górniak 1988, Coelho et al. 2007).

Diversas substâncias têm sido testadas como antídoto contra a intoxicação por MF, em especial, em ratos e camundongos. Dentre essas, a acetamida $\left(\mathrm{CH}_{3} \mathrm{CONH}_{2}\right)$ tem demonstrado considerável efeito protetor. De fato, quando administrada a ratos, previamente à intoxicação por algumas PBCMS (P. marcgravii, P. jururana, A. bilabiata, Pseudocalymma elegans, A. rigida, A. exotropica, M. pubiflora) ou MF, a acetamida evita o desenvolvimento dos sinais clínicos e a morte dos animais (Peixoto et al. 2011b). Convém dizer que o efeito protetor da acetamida já havia sido demonstrado em ratos intoxicados com P. marcgravii e por MF (Gór- 
niak et al. 1994), entretanto, curiosamente, esses autores não mencionaram em suas conclusões que MF seria o princípio tóxico determinante da morte dos animais intoxicados por essa planta. Efeito protetor semelhante foi recentemente descrito em ovinos, caprinos e coelhos intoxicados por P. elegans (Helayel et al. 2011). Além disso, verificou-se a eficácia da utilização desse antídoto em cobaios e ovinos intoxicados por Dichapetalum cymosum, uma importante planta africana que contêm MF como princípio tóxico (Egyed \& Schultz 1986). Em relação aos bovinos, há relatos de que a acetamida não seria eficaz na reversão da intoxicação por P. marcgravii (Soto-Blanco et al., 2004).

Ainda não foi comprovado, portanto, se a administração de acetamida é capaz de evitar o aparecimento dos sinais clínicos e a morte de bovinos intoxicados com P. marcgravii ou por MF, ou seja, se esse composto é, de fato, o responsável pela morte dos ruminantes que ingerem e se intoxicam espontaneamente por essas plantas. Tal demonstração comprovaria de forma prática que esse composto é o princípio tóxico determinante da morte dos bovinos intoxicados por P. marcgravii.

Essa comprovação, além do seu significado diagnóstico, pode ter grande relevância econômica na pecuária, uma vez que estudos genéticos com o objetivo de capacitar bactérias ruminais a metabolizar MF, tornando-o inócuo, têm sido desenvolvidos na Austrália (Gregg et al. 1998). No Brasil, esses estudos encontram-se em fase inicial de desenvolvimento pelo intitulado Projeto Milênio coordenado pelo Dr. Franklin Riet-Correa ${ }^{8}$. Tal condição possibilitaria a profilaxia da intoxicação natural por PBCMS, uma vez que, em termos práticos, o tratamento é inviável através da utilização de antídotos, como a própria acetamida, dada a rapidez da evolução clínica.

\section{MATERIAL E MÉTODOS}

Foram utilizadas cinco vacas adultas, com pesos entre 292 e $465 \mathrm{~kg}$, mestiças de Holandês, soropositivas para brucelose, sem alterações clínicas, procedentes da Pesagro, RJ e que, deveriam ser eutanasiadas para atender à legislação brasileira.

Utilizou-se monofluoroacetato AMF) (Sigma-Aldrich Co) ${ }^{9}$, com grau de pureza $\geq 95 \%$, pertencente ao lote 23207046 . A dose de MF administrada aos bovinos foi estabelecida com base na $\mathrm{LD}_{50}$ descrita por Humphreys (1988). A acetamida $\left(\mathrm{CH}_{3} \mathrm{CONH}_{2}\right)$, $\mathrm{PA}=59,07$ (Proquímios) $^{10}$, empregada nesse estudo pertencia ao lote $08 / 107$. As doses administradas aos bovinos foram determinadas tendo como parâmetro os estudos pioneiros de Egyed \& Schultz (1986). As doses de MF, bem como de acetamida fornecida aos bovinos foram previamente pesadas em balança eletrônica de precisão e diluídas, no momento de sua administração, em $50 \mathrm{~mL}$ e $500 \mathrm{~mL}$ a $2 \mathrm{~L}$ de água destilada, respectivamente, conforme demonstrado no Quadro 1. Palicourea marcgravii utilizada nos experimentos (folhas jovens e maduras) foi coletada no município de Valença, Rio de Janeiro, em 14, 21 e 22 abr. 2009, acondi-

\footnotetext{
${ }^{8}$ Franklin Riet-Correa, Laboratório de Anatomia Patológica, Centro de Saúde e Tecnologia Rural (CSTR), Universidade Federal de Campina Grande (UFCG), Campus de Patos, Santa Cecília, Patos, PB 58700-000, Brasil.

9 Sigma-Aldrich, 3050 Spruce Street, St Louis, MO 63103, USA <http:// www.sigma-aldrich.com>

${ }^{10}$ Proquímios, Rua Coronel Tamarindo 3010, Bangu, Rio de Janeiro, RJ 21848-440, Brasil, <http://www.proquimios.com.br>
}

cionada em sacos de pano e mantidas em geladeira a $4^{\circ} \mathrm{C}$, por no máximo, 24 horas.

Os experimentos e as necropsias foram realizados entre abril e maio de 2009 no Setor de Anatomia Patológica do Projeto Sanidade Animal Embrapa/UFRRJ, município de Seropédica, RJ. Os bovinos foram mantidos em baias individuais de alvenaria com área de $4,0 \mathrm{~m}^{2}$ e piso de cimento, com acesso à água e capim picado (Panicum maximum) à vontade. 0 processamento do material coletado durante as necropsias, assim como a confecção de lâminas para o exame histológico, foram realizados no Laboratório de Histopatologia do Setor acima mencionado.

0 experimento piloto, denominado de auto-direcional, foi realizado em três animais, com o objetivo de utilizar o menor número possível destes, por se tratar de um estudo descritivo. Os experimentos subsequentes foram executados de forma sucessiva, na dependência dos primeiros resultados obtidos.

Três bovinos foram receberam monofluoroacetato (Sigma-Aldrich Co), por via oral, na dose de $0,5 \mathrm{mg} / \mathrm{kg}$ e, em seguida, a dois desses animais administraram-se acetamida, por via oral (sonda orogástrica), nas doses de 0,38 e $2,0 \mathrm{~g} / \mathrm{kg}$. Outros dois bovinos receberam manualmente na boca, folhas frescas de $\boldsymbol{P}$. marcgravii na dose de $1,0 \mathrm{~g} / \mathrm{kg}$, sendo que um deles recebeu, em seguida, 1,0g/kg de acetamida, por via oral, conforme apresentado no Quadro 1. Os animais intoxicados por MF ou P. marcgravii, cuja administração de acetamida preveniu os sinais clínicos e o óbito, receberam novamente, após uma semana, a mesma dose de MF ou P. marcgravii anteriormente utilizada, porém sem acetamida. Desta forma, os animais cuja acetamida foi capaz de induzir efeito protetor, serviram como controle deles mesmos, o que evitou a ocorrência de possíveis variações individuais, além de permitir a utilização de um menor número de animais nos experimentos.

Os animais foram observados continuamente e submetidos a exames clínicos, no mínimo, a cada duas horas, após o começo do experimento até a morte, exceto nos animais cuja administração de acetamida evitou o aparecimento dos sinais clínicos e do óbito, nesses casos, os animais foram acompanhados clinicamente por 72 horas. Durante o exame clínico era realizada a avaliação dos seguintes parâmetros clínicos: frequências cardíaca e respiratória, presença ou não de pulso venoso, bem como a postura e o comportamento dos animais. Foram observados ainda, a ingestão de água e capim, além da frequência da micção e defecação dos animais no decorrer do experimento. Quando os animais começaram a manifestar sinais clínicos, a frequência dos exames clínicos foi intensificada.

Todos os bovinos foram necropsiados imediatamente após a morte. Fragmentos de diversos órgãos foram coletados, fixados em formol $10 \%$, processados rotineiramente para histopatologia e corados pela Hematoxilina e Eosina.

\section{RESULTADOS}

Os principais dados sobre o delineamento experimental e o desfecho encontram-se no Quadro 1.

\section{Efeito protetor da acetamida}

A acetamida administrada imediatamente após a intoxicação por monofluoroacetato $(0,5 \mathrm{mg} / \mathrm{kg})$ ou folhas frescas de Palicourea marcgravii $(1,0 \mathrm{~g} / \mathrm{kg})$, evitou o aparecimento dos sinais clínicos e a morte de todos os animais, exceto no Bovino 235, que foi intoxicado com $0,5 \mathrm{mg} / \mathrm{kg}$ de $\mathrm{MF}$ e, mesmo após receber $0,38 \mathrm{~g} / \mathrm{kg}$ de acetamida, manifestou sinais clínicos e morreu. 


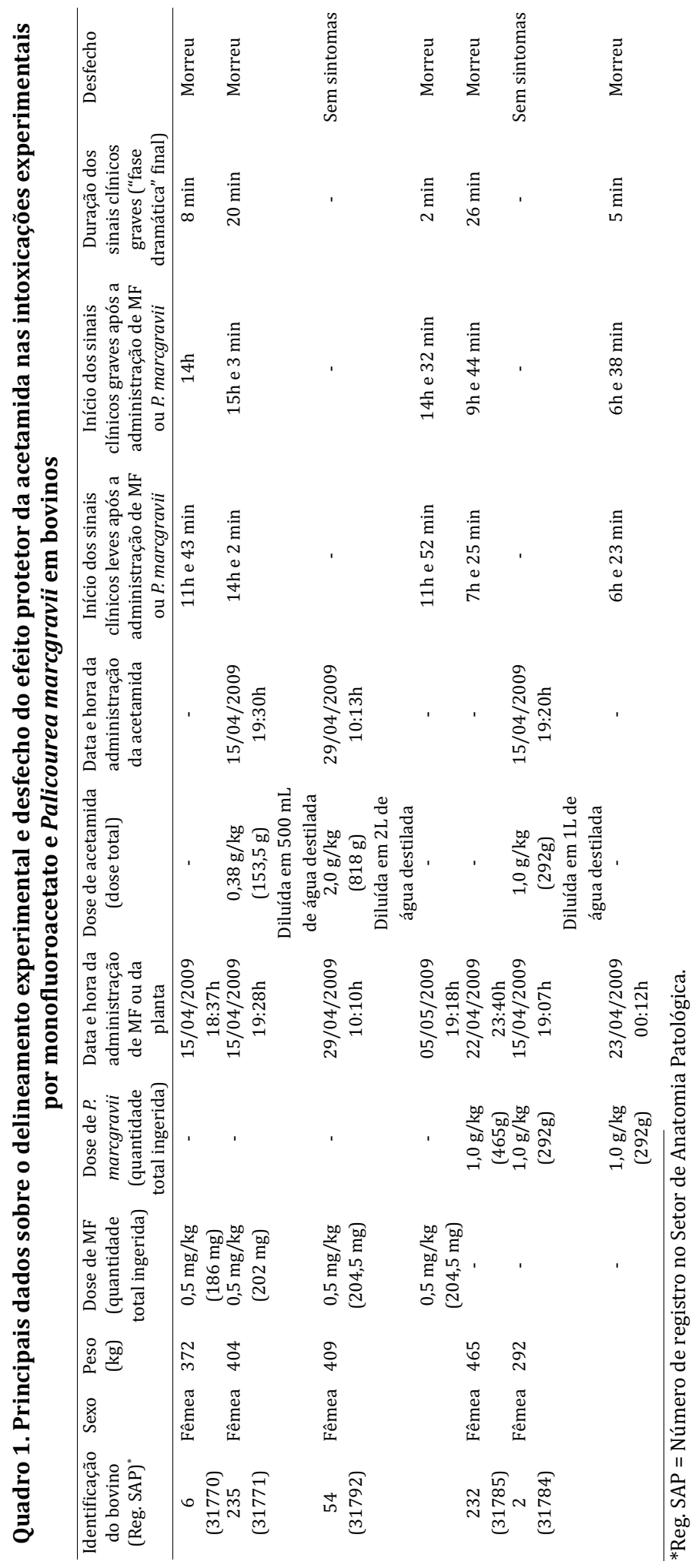

Tal efeito protetor foi, de fato, confirmado após uma semana, quando o mesmo protocolo experimental foi repetido, para cada bovino, porém sem a administração de acetamida. Nessa etapa, todos os bovinos manifestaram sinais clínicos e morreram. Desta maneira, descartamos eventuais variações individuais quanto à sensibilidade dos bovinos ao MF ou P. marcgravii.
Início dos sinais clínicos e duração dos sinais clínicos leves e graves

Nos experimentos em que a acetamida não foi administrada, todas as vacas intoxicadas por monofluoroacetato $(0,5 \mathrm{mg} / \mathrm{kg})$ ou pelas folhas frescas de P. marcgravii $(1,0 \mathrm{~g} / \mathrm{kg})$ apresentaram sinais clínicos e morreram.

O tempo decorrido entre a administração de MF e a manifestação dos primeiros sinais clínicos leves variou entre 11h43min e 14h2min; já os sinais clínicos graves, se iniciaram entre $14 \mathrm{~h}$ e $15 \mathrm{~h} 3 \mathrm{~min}$ após a intoxicação por MF. Nos experimentos realizados com $\boldsymbol{P}$. marcgravii $(1,0 \mathrm{~g} / \mathrm{kg})$, os primeiros sintomas leves foram observados de $6 \mathrm{~h} 23 \mathrm{~min}$ a $7 \mathrm{~h} 25 \mathrm{~min}$ e os graves de $6 \mathrm{~h} 38 \mathrm{~min}$ a $9 \mathrm{~h} 44 \mathrm{~min}$. A duração dos sinais clínicos graves ("fase dramática") variou de 2 a $20 \mathrm{~min}$, nos animais intoxicados por MF, e de 5 a $26 \mathrm{~min}$, nos experimentos com P. marcgravii.

\section{Quadro clínico geral}

Nesse estudo, o quadro clínico manifestado pelos bovinos intoxicados por monofluoroacetato (Bovinos 6, 54 e 235) correspondeu totalmente ao observados nos animais intoxicados por P. marcgravii (Bov. 2 e 232), e caracterizou-se, em geral, por taquicardia, taquipnéia, tremores musculares, jugular repleta (Fig.1) com pulso venoso positivo, instabilidade, incoordenação, ligeira perda de equilíbrio, por vezes cambaleavam, apoiavam a cabeça no flanco (postura de auto-auscultação), inquietação (levantavam e deitavam repetidamente). Quatro animais tiveram arritmia (Bov. 2, 54, 232 e 235), três midríase (Bov. 6, 232 e 235), polaquiúria (Bov. 6, 54 e 235) e apatia (Bov. 6, 54 e 232). Observou-se ainda em dois animais veias da face ingurgitadas (Bov. 235 e 232). Na "fase final" da intoxicação, todos os animais deitavam-se e levantavam-se com maior frequencia, deitavam ou caíam (Fig.2) em decúbito lateral, esticavam os membros, faziam movimentos de pedalagem, apresentavam hiperpneia (respiração ofegante), opistótono, nistagmo, por vezes, mugidos e morriam em poucos minutos.

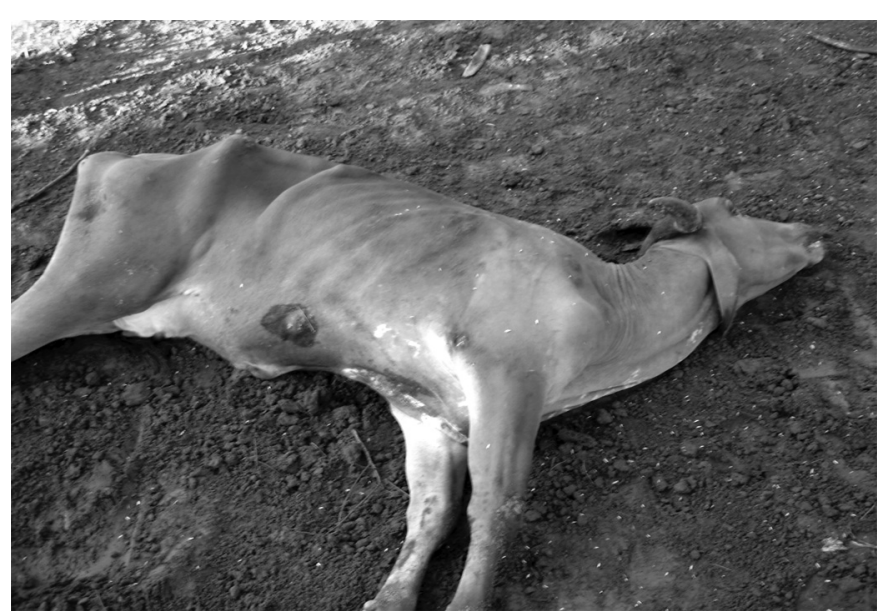

Fig.1. Intoxicação experimental com folhas frescas de Palicourea marcgravii (1,0g/kg). Bovino 2 em decúbito lateral esquerdo, com opistótono e a veia jugular acentuadamente ingurgitada, na "fase final" da intoxicação. 


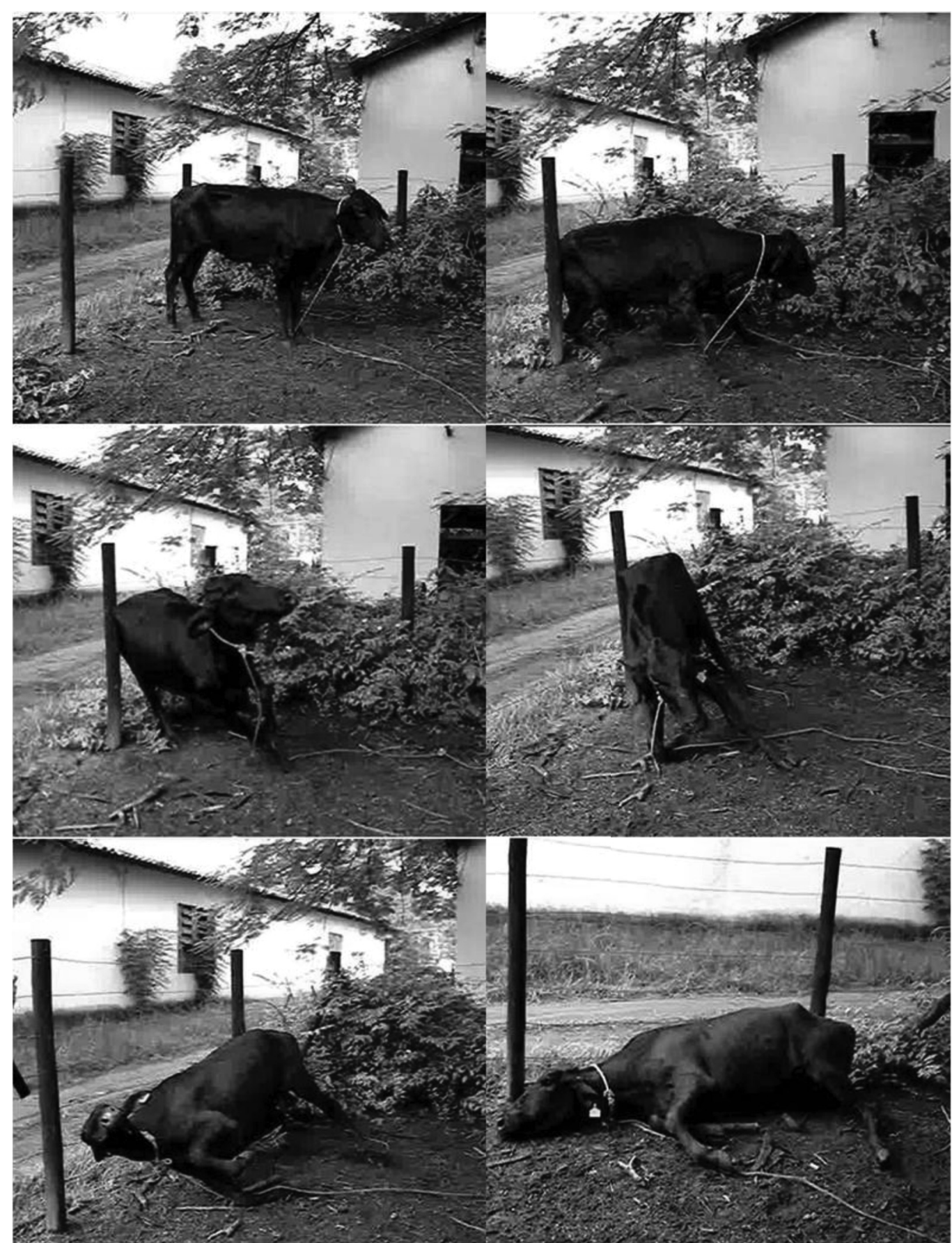

Fig.2. Intoxicação experimental com monofluoroacetato de sódio $(0,5 \mathrm{mg} / \mathrm{kg})$. Sequência de fotos evidenciando perda do equilíbrio e queda súbita ao solo. Bovino 6 no início da "fase dramática" final da intoxicação.

\section{Achados de necropsia}

A necropsia dos bovinos intoxicados por P. marcgravii e monofluoroacetato, em geral, evidenciou, veias jugulares, aurículas, veias cava cranial e caudal, ázigos, costo cervical e pulmonares leve a moderadamente ingurgitadas. Havia raras equimoses subepicárdicas no ventrículo direito (próximo as coronárias) e no ápice do ventrículo esquerdo (Bov. 6), bem como moderada quantidade de líquido espumoso róseo da traquéia aos brônquios (Bov. 232). Três animais (Bov. 2, 6 e 54) apresentaram leve a acentuado edema da subserosa da vesícula biliar, sobretudo, na sua inserção no fígado; no Bovino 2, verificou-se também, leve edema por extensão entre pâncreas e duodeno. Os demais achados constituíram-se de discretas petéquias na mucosa da traquéia (Bov. 6), leve a moderada congestão pulmonar (Bov. 6 e 232), renal (Bov. 235) e da mucosa da vesícula biliar (Bov. 6), leve hidropericárdio (Bov. 54) e áreas enfisematosas nos bordos dos lobos craniais e caudas do pulmão (Bov. 235). Observaram-se ainda nos dois animais (Bov. 2 e 232) intoxicados com folhas frescas de P. marcgravii $(1,0 \mathrm{~g} / \mathrm{kg})$, 
fragmentos reconhecíveis das folhas da planta no rúmen (Fig.3). Nos demais órgãos não foram encontradas alterações dignas de nota.

\section{Achados histopatológicos}

0 exame histopatológico evidenciou, no rim de todos os bovinos leve (Bov. 2 e 54) a acentuada (Bov. 6 e 235) degeneração hidrópico-vacuolar das células epiteliais dos túbulos uriníferos contornados distais (TCD) e, por vezes, dos túbulos retos (Bov. 232 e 235), associada à picnose nuclear (Fig.4). Alguns TCD exibiam diferentes estágios da mesma lesão (incipiente e avançada). Havia ainda, leve (Bov. 2) a moderada (Bov. 235) congestão, leve infiltrado inflamatório linfoplasmocitário intersticial (Bov. 2, 6 e 235), leve dilata- ção da cápsula de Bowman (Bov. 6) e filtrado glomerular em forma de glóbulos (Bov. 6, 232 e 235). No fígado, em geral, observaram-se leve a moderada congestão, necrose de coagulação focal ou individual aleatória, discreta a moderada tumefação e moderada vacuolização de hepatócitos, predominantemente, centrolobular e discreto a moderado infiltrado inflamatório linfoplasmocitário periportal, bem como esférulas eosinofílicas nos sinusóides hepáticos (corpúsculos de choque) e dilatação dos sinusóides, especialmente, da região centrolobular. No coração observaram-se fibras musculares individuais ou grupos de cardiomiócitos com aumento da eosinofilia sarcoplasmática, perda das estriações e, por vezes, núcleos picnóticos (Bov. 6, 232 e 235). Nos demais órgãos não foram encontradas alterações significativas.

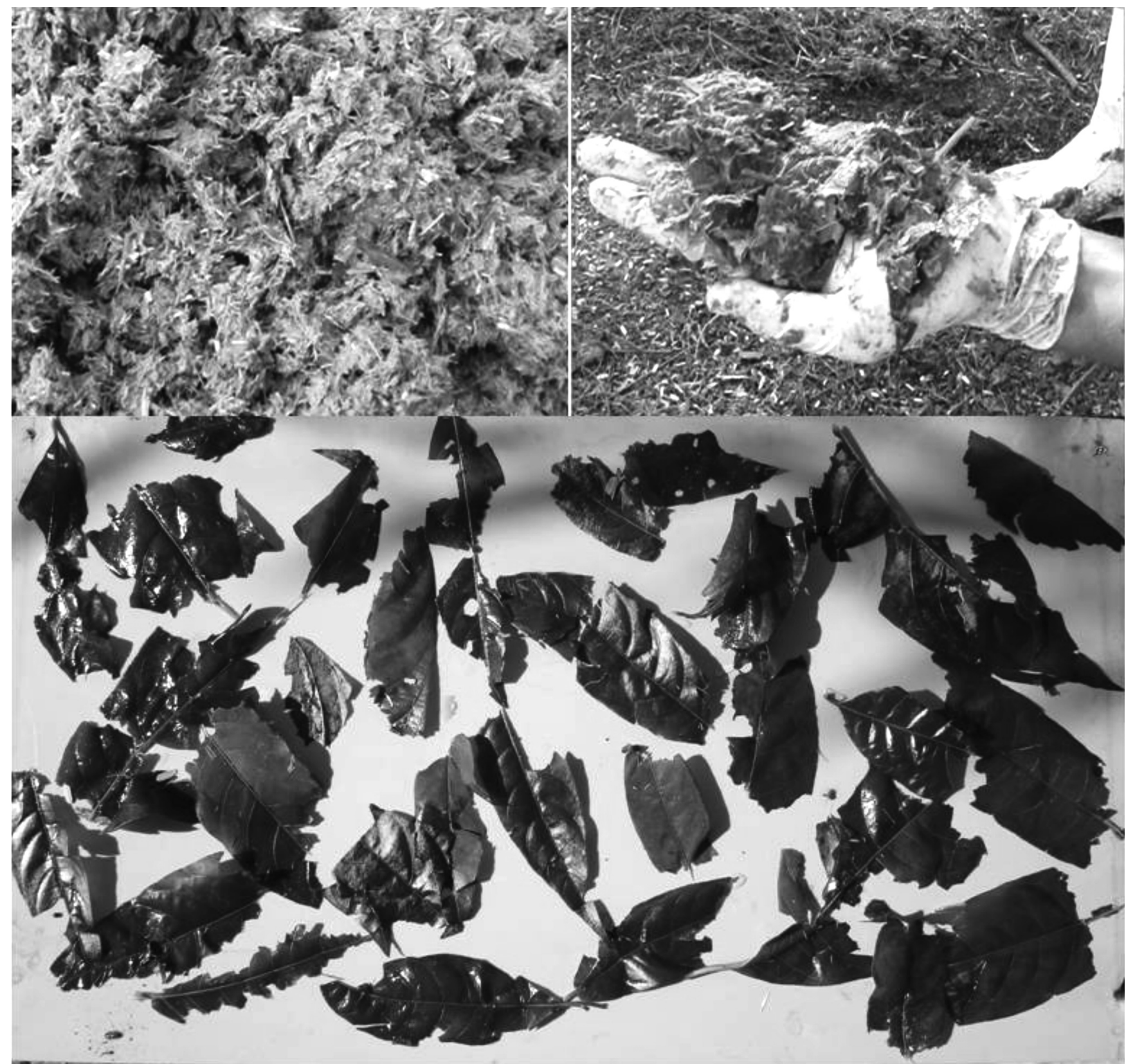

Fig.3. Intoxicação experimental com folhas frescas de Palicourea marcgravii (1,0g/kg). Fragmentos de folhas da planta, ainda reconhecíveis, recuperados do rúmen durante a necropsia do Bovino 232. 


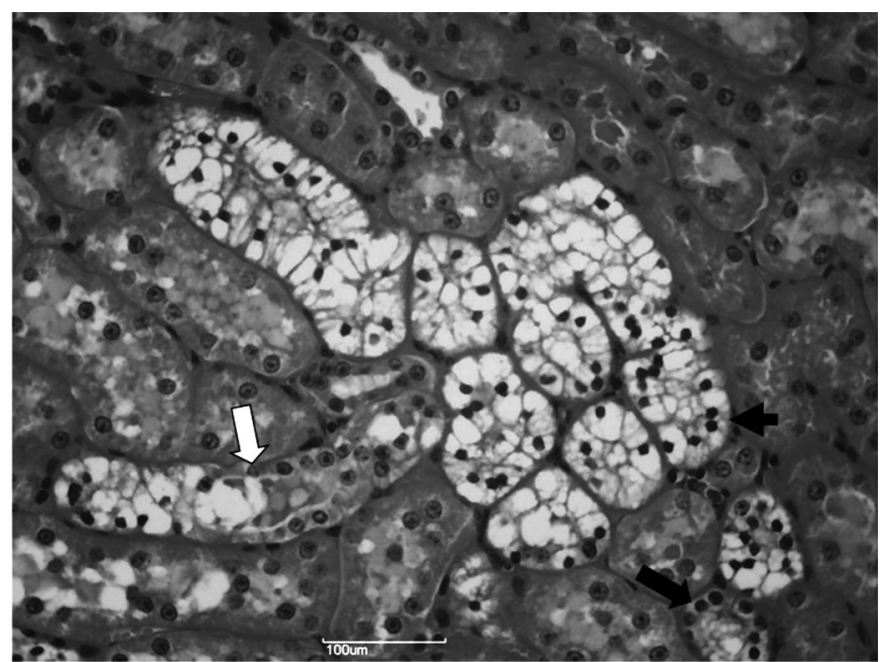

Fig.4. Degeneração hidrópico-vacuolar das células epiteliais dos túbulos uriníferos contornados distais associada à picnose nuclear (Seta preta: lesão incipiente. Cabeça da seta: lesão mais avançada, caracterizada por marcada picnose nuclear). Notar que um túbulo exibe diferentes estágios da mesma lesão (incipiente e avançada) (Seta branca). HE, obj.20x. Bovino 6 (SAP 31770) intoxicado experimentalmente com monofluoroacetato $(0,5 \mathrm{mg} / \mathrm{kg})$.

\section{DISCUSSÃO}

Neste estudo, demonstrou-se que a acetamida, quando administrada em quantidades suficientes, minutos após os bovinos receberem, por via oral, monofluoroacetato $(0,5 \mathrm{mg} /$ $\mathrm{kg}$ ) ou Palicourea marcgravii $(1,0 \mathrm{~g} / \mathrm{kg})$, exerce efeito protetor idêntico para ambos os agentes, uma vez que evitou o aparecimento dos sinais clínicos e a morte de todos os animais. A acetamida, dessa forma, pode ser considerada um antídoto eficaz para ambas as intoxicações, o que indica que monofluoroacetato (MF) é o princípio tóxico desta planta. Tal efeito protetor foi, de fato, confirmado após uma semana, quando o mesmo protocolo experimental foi repetido, para cada bovino, porém sem a administração de acetamida; nesta etapa, todos os bovinos manifestaram sinais clínicos e morreram subitamente. 0 emprego dessa metodologia possibilitou que os animais intoxicados por MF ou P. marcgravii servissem como controle deles mesmos, o que descartou a ocorrência de possíveis variações individuais (sensibilidade) e valorizou os resultados obtidos, além de permitir a utilização de um menor número de animais nos experimentos.

Esses resultados foram semelhantes aos recentemente descritos por Helayel et al. (2011), os quais demonstraram que a administração prévia acetamida também possui efeito antagônico protetor em ovinos, caprinos e coelhos que receberam experimentalmente Pseudocalymma elegans, planta que também causa morte súbita. Adicionalmente, estudos complementares realizados em ratos confirmaram que a acetamida, quando administrada previamente, evita tanto a manifestação dos sinais clínicos, quanto à morte dos ratos que ingeriram folhas frescas de Palicourea marcgravii ou os extratos concentrados de P. marcgravii, P. juruana, Pseudocalymma elegans, Arrabidaea bilabiata, Mascagnia pubiflora, Amorimia rigida e A. exotropica, e por MF
(Peixoto et al. 2011b). Convém dizer que o efeito protetor da acetamida já havia sido anteriormente demonstrado em ratos que receberam extrato aquoso de $P$. marcgravii e MF; no entanto, neste caso, a acetamida foi administrada por via intraperitoneal (Górniak et al. 1994) e, curiosamente, esses autores não concluiram que o MF é o princípio tóxico determinante da morte dos animais. É importante mencionar que o trabalho pioneiro, com a finalidade de avaliar a eficácia da acetamida em casos de intoxicação por planta que contém MF, foi desenvolvido na África do Sul por Egyed \& Schultz (1986), em cobaios e ovinos intoxicados por Dichapetalum cymosum.

Por outro lado, há relatos na literatura de que a acetamida não é eficaz na reversão da intoxicação por $P$. marcgravii em bovinos (Soto-Blanco et al. 2004). Neste ponto cabem algumas considerações. Egyed \& Schultz (1986) já mencionavam que a eficácia da acetamida depende de diversos fatores, como a toxidez da planta em questão, tempo decorrido entre a intoxicação e fornecimento do antídoto, doses de acetamida e da planta administradas. Ao que parece, algum desses fatores pode justificar, pelo menos em parte, essa divergência descrita por Soto-Blanco et al. (2004) e aos achados verificados nesse estudo. Além disso, é óbvio que a eficácia da acetamida na reversão da intoxicação por $P$. marcgravii ou MF, isto é, após início dos sinais clínicos, é sem dúvida muito menor do que sua capacidade de evitar ou prevenir essas intoxicações, quando sua administração é feita de forma prévia ou imediatamente após a administração dos compostos tóxicos, seja a planta ou MF. Em ratos, entretanto, já foi demonstrado que a reversão da intoxicação por folhas frescas P. marcgravii $(4,0 \mathrm{~g} / \mathrm{kg})$ é, por vezes, possível mesmo após o animal manifestar sintomas graves ( 2 horas após o consumo da planta) através do tratamento com 4,0g/ $\mathrm{kg}$ de acetamida (Peixoto et al. 2011b). Ao que tudo indica, essa reversão, só se verifica em animais que apresentam quadro mais protraído, com evolução clínica mais longa. Portanto, embora seja verdade que a acetamida não é eficaz na reversão do quadro de intoxicação por P. marcgravii - não há tempo hábil, pela evolução de poucos minutos - ela o é, na prevenção da intoxicação, quando administrada previamente em quantidades suficientes.

Não obstante, algumas considerações devem ser feitas em relação ao Bovino 235 que, apesar de ter recebido acetamida $(0,38 \mathrm{~g} / \mathrm{kg})$, logo após intoxicação por MF $(0,5 \mathrm{mg} / \mathrm{kg})$ manifestou sinais clínicos e morreu. Tudo indica que, neste caso a quantidade de acetamida administrada (menor dose utilizada nos experimentos) foi insuficiente para antagonizar a quantidade de MF. Esse achado demonstra claramente que a eficácia da acetamida é dose-dependente como postulam Egyed \& Schultz (1986). Também corroboram com esta hipótese resultados similares descritos por outros autores, cuja administração de baixas doses de acetamida (quantidades insuficientes) e/ou doses elevadas de plantas brasileiras que causam morte súbita (PBCMS) ou MF, não foram capazes de evitar o aparecimento dos sinais clínicos e/ou morte de ovinos, caprinos (Helayel et al. 2011) e ratos (Peixoto et al. 2011b), nestes experimentos, contudo, após ajuste da dose de acetamida e/ou da PBCMS utilizada, o efeito protetor da acetamida também pode ser evidenciado. 
Antes da comparação e demonstração do efeito protetor da acetamida nas intoxicações por $P$. marcgravii e MF em bovinos, observada neste estudo, já havia fortes evidências circunstanciais de que MF seria a causa, ou pelo menos um dos compostos importantes envolvidos na gênese e no desfecho das intoxicações por PBCMS. De fato, recentemente demonstrou-se que o quadro clínico-patológico manifestado por bovinos (Nogueira et al. 2010) e ovinos (Peixoto et al. 2010) intoxicados experimentalmente com MF corresponde, em diversos aspectos, ao observado em casos de intoxicação por PBCMS e, que, a típica degeneração hidrópico-vacuolar (DHV) dos túbulos contornados distais (TCD) associada à picnose nuclear, verificada no rim de bovinos intoxicados natural e experimentalmente com todas as PBCMS (Tokarnia et al. 2000, Barbosa et al. 2003, Oliveira et al. 2004, Helayel et al. 2009) é uma lesão característica da intoxicação por MF em bovinos (Nogueira et al. 2010) e ovinos (Peixoto et al. 2010). Além disso, diversas outras substâncias que já foram isoladas dessas plantas, em especial, de P. marcgravii, como alcalóides, saponinas, ácidos málico, palicúrico e mioctônico, salicilato de metila, cristais de oxalato de cálcio, tanoides, ácidos salicílico e o-metoxibenzóico, cafeína, alcalóides N-metiltiramina e 2 -metiltetrahidro- $\beta$-carbolina (Guimarães 1934, Mello \& Fernandes 1940, Barnes \& Gilbert 1960, Cascon \& Mors 1962, Peckolt 1868, Gagnin \& Maravalhas 1969, Górniak 1988, Morita et al. 1989, Kemmerling 1996, Coelho et al. 2007) não induzem o quadro clínico-patológico de "morte súbita" e, muito menos à DHV. Outros autores, contudo, são da opinião que MF não seria o princípio determinante das mortes dos animais que ingerem essas plantas (Habermehl 1986, González et al. 2000) ou que haveria outros compostos que poderiam causar a morte dos animais (Guimarães 1934, Hoehne 1939, Mello \& Fernandes 1940, Peckolt 1868, Corrêa 1969, Górniak et al. 1986), ter efeito sinérgico com MF (Kemmerling 1996) ou ainda contribuir para a toxicidade da planta (Górniak 1988, Coelho et al. 2007).

Nesse estudo, a intoxicação por folhas frescas de P. marcgravii ou MF, nos experimentos em que a acetamida não foi administrada aos bovinos, provocaram quadros clínico-patológicos semelhantes, caracterizados por "morte súbita", isto é, uma intoxicação de evolução superaguda. Esses achados corresponderam, em diversos aspectos, ao observado nos casos de intoxicação por PBCMS (Tokarnia et al. 2000).

No presente estudo, o exame histopatológico revelou, em todos os bovinos, o aparecimento da típica DHV dos TCD associada à cariopicnose, lesão idêntica à descrita por Döbereiner \& Tokarnia (1959) no rim de bovinos intoxicados por P. marcgravii. Mais tarde esta lesão também foi observada no rim de bovinos intoxicados natural e experimentalmente com todas as outras PBCMS (Tokarnia et al. 2000, Barbosa et al. 2003, Oliveira et al. 2004, Helayel et al. 2009) e recentemente na intoxicação por MF em bovinos (Nogueira et al. 2010), ovinos (Peixoto et al. 2010) e ratos (Peixoto et al. 2011b). A vacuolização de hepatócitos observada nesse estudo pode ou não ser associada ao efeito direto do MF, pois não se pode descartar que esta lesão seja resultado da anóxia determinada pela insuficiência cardíaca prévia à fase dramática da morte súbita (Nogueira et al. 2010). Achados similares foram descritos em casos de intoxicação de bovinos, por MF (Nogueira et al. 2010) e diversas PBCMS (Döbereiner \& Tokarnia 1982, Tokarnia et al. 1969, 1990, 2004, Consorte et al. 1994, Oliveira et al. 2004, Helayel et al. 2009). No coração de três dos cinco bovinos que receberam MF ou P. marcgravii, verificaram-se fibras musculares individuais ou grupos de cardiomiócitos com aumento da eosinofilia sarcoplasmática, perda das estriações e, por vezes, núcleos picnóticos. Este achado pode estar relacionado aos efeitos tóxicos diretos ou indiretos do MF, entretanto, é mais provável que esta lesão seja secundária a depleção dos níveis de ATP e a hipóxia do miocárdio. De fato, estudos eletrocardiográficos em gatos intoxicados por MF demonstraram arritmias ventriculares e alterações inespecíficas de repolarização ventricular, tais como supradesnível de ST e aumento da onda T, o que sugere lesões de hipóxia do miocárdio (Collicchio-Zuanaze \& Andrade 2011). Convém dizer que em outros estudos conduzidos em bovinos, ovinos, caprinos, coelhos e ratos intoxicados por MF ou outras PBCMS tais lesões regressivas tem sido observadas com certa frequência (Peixoto, dados não publicados) e que, estudos imuno-histoquímicos deverão ser realizados para permitir uma melhor interpretação desse achado.

Embora a comercialização do MF seja proibida no país (Adesp 2007), sabe-se que este composto ainda é ilegalmente comercializado por ambulantes (Apevisa 2009) e que, se armazenado sob condições adequadas, a sua toxidez é mantida por décadas (Eisler 1995). Recente prova disso ocorreu na Fundação Parque Zoológico de São Paulo, onde pelo menos 73 animais de diversas espécies morreram intoxicados, de forma criminosa, por esse composto segundo a perícia (Ortis 2005), e em seres humanos no Estado do Rio Grande do Sul em 2005-2009, onde foram confirmados 17 casos de intoxicação por MF (Nicolella et al. 2005, 2006, 2007, 2008/2009).

Em relação à questão da nomenclatura dos rodenticidas de uso legalizado ou não no Brasil, cabem algumas considerações. É importante ressaltar que os médicos veterinários devem ficar atentos, uma vez que a bromadiolona (bromadiolone) é comercialmente encontrada com o nome Mão Branca ${ }^{\circ}$, que é o nome comercial original de outro famoso raticida ilegal no país, o MF. O clínico, contudo, deve ter conhecimento deste fato, que pode gerar confusões, e saber distinguir o Mão Branca® falso (bromodiolona, um anticoagulante, peletizado cor de rosa) do Mão Branca® original (MF, líquido azul). Além disso, é importante lembrar que não é rara a intoxicação criminosa pelo $\mathrm{MF}$, associado a outros raticidas para aumentar o poder letal, como por exemplo, a associação com carbamato aldicarb ("chumbinho") e anticoagulantes (Collicchio-Zuanaze \& Andrade 2011), o que obviamente altera o quadro clínico e pode dificultar o diagnóstico.

O presente estudo demonstra a eficácia da utilização concomitante da acetamida na prevenção da morte de bovinos que ingeriram MF, o que corrobora com os resultados já relatados, especialmente, em ratos (Górniak et al. 1994, Peixoto et al. 2011b). Desta forma, a utilização da 
acetamida na tentativa do tratamento de animais (sobretudo, naqueles cujo quadro clínico de intoxicação for mais protraído) ou seres humanos intoxicados por MF deve ser realizada sempre que possível. Em relação aos bovinos, todavia, o emprego da acetamida no tratamento da intoxicação por PBCMS, deve ser vista com cautela, uma vez que, para ser eficaz, este composto deve ser administrado aos animais poucas horas antes ou imediatamente após o animal ingerir a planta, o que, em termos práticos, é inviável, para a grande maioria dos casos, dada a rápida evolução clínica, bem como pelo alto valor comercial deste antídoto e sua difícil aquisição e disponibilidade no campo. Apesar disso, outros autores sugerem um "potencial tratamento racional" da intoxicação por P. marcgravii em bovinos, através da administração de acetamida (Górniak et al. 1994).

Convém ressaltar que o presente estudo não teve como objetivo testar a eficiência de potenciais antídotos com o intuito de instituir tratamentos, muito menos, sugerir ou difundir a sua utilização em casos de bovinos intoxicados por PBCMS, e sim comprovar de forma prática que o MF é o princípio tóxico de $P$. marcgravii responsável pelos sinais clínicos e a morte dos animais, com a finalidade de fornecer subsídios para o desenvolvimento de estudos no Brasil que envolvam a metabolização de MF por bactérias ruminais, o que teria grande aplicabilidade econômica, uma vez que pelo menos 500.000 bovinos morrem, anualmente, intoxicados por essa planta (Tokarnia et al. 2002). Cabe dizer que, pesquisadores australianos modificaram geneticamente a bactéria ruminal Butyrivibrio fibrisolvens, através da introdução de um gene isolado de Moxarella sp., que codifica uma dehalogenase, capaz de hidrolizar o MF tornando-o inócuo (Gregg et al. 1994, 1998). No Brasil, pesquisadores envolvidos no Projeto Milênio isolaram nove bactérias (duas ruminais e sete do solo, próximo a plantas que contêm MF), que hidrolizam o MF, as quais serão na próxima etapa deste projeto, inoculadas no rúmen de caprinos no intuito de avaliar se tais bactérias conferem a resitência desejada aos animais que ingerem plantas que contêm MF (Riet-Correa, comunicação pessoal). A implementação dessa tecnologia no rebanho bovino brasileiro seria uma técnica profilática viável contra a intoxicação por plantas que contêm o MF como princípio ativo.

\section{REFERÊNCIAS}

Adesp 2007. Associação de Empresas Controladoras de Pragas do Estado de São Paulo. Disponível em <http://www.adesp.org.br> Acesso 7 jul. 2007.

Apevisa 2009. Apevisa apreende 302 frascos com raticida ilegal. Agência Pernambucana de Vigilância Sanitária (Apevisa). Disponível em <http:// www.saude.pe.gov.br/noticias.php?codigo $=1066 \&$ pagina $=2 \&$ pu-blicar=1> Acesso 20 fev. 2009.

Barbosa J.D., Oliveira C.M.C., Tokarnia C.H. \& Riet-Correa F. 2003. Comparação da sensibilidade de bovinos e búfalos à intoxicação por Palicourea marcgravii (Rubiaceae). Pesq. Vet. Bras. 23(4):167-172.

Barnes R.A. \& Gilbert M.E.A. 1960. Investigação química preliminar de várias plantas brasileiras: Presença de alcalóides, saponinas e outras substâncias. Bolm Inst. Quím. Agric., Rio de J., 58:9-26.

Cascon C.S \& Mors W.B. 1962. Substâncias isoladas da Palicourea marcgravii St. Hil: uma nova síntese da N-metil-tiramina. Anais Assoc. Bras. Quim. 21:53-60.
Coelho E.G., Amaral A.C.F., Ferreira J.L.P., Santos A.G., Pinheiro M.L.B. \& Silva J.R.A. 2007. Calcium oxalate crystals and methyl salicylate as toxic principles of the fresh leaves from Palicourea longiflora, an endemic species in the Amazon state. Toxicon 49:407-409.

Collicchio-Zuanaze R.C. \& Andrade S.F. 2011. Rodenticidas, p.171-194. In: Nogueira R.M.B. \& Andrade S.F. (Eds), Manual de Toxicologia Veterinária. Roca, São Paulo. 323p.

Consorte L.B., Peixoto P.V. \& Tokarnia C.H. 1994. Intoxicação experimental por Pseudocalymma elegans (Bignoniaceae) em ovinos. Pesq. Vet. Bras. 14(4):123-133.

Corrêa P.M. 1969. Dicionário das Plantas Úteis do Brasil e das Exóticas Cultivadas. Vol.4. Instituto Brasileiro do Desenvolvimento Florestal (IBDF), Rio de Janeiro. 765p.

Cunha L.C. 2008. Avaliação dos efeitos tóxicos de Mascagnia rigida em ratos: estudo anatomopatológico, comparação entre metodologias cromatográficas para detecção do fluoroacetato de sódio. Dissertação de Mestrado em Patologia Experimental e Comparada, Universidade de São Paulo, São Paulo, SP. 100p.

Döbereiner J. \& Tokarnia C.H. 1959. Intoxicação de bovinos pela "erva-de-rato" (Palicourea marcgravii St. Hil.) no vale do Itapicuru, Maranhão. Arqs Inst. Biol. Anim., Rio de J., 2:83-91.

Döbereiner J. \& Tokarnia C.H. 1982. Intoxicação experimental por Palicourea grandiflora (Rubiaceae) em coelhos. Pesq. Vet. Bras. 2(3):121-124.

Egyed M.N. \& Schultz R.A. 1986. The efficacy of acetamide for the treatment of experimental Dichapetalum cymosum (gibflaar) poisoning in sheep. Onderstepoort J. Vet. Res. 54:231-234.

Eisler R. 1995. Sodium monofluoroacetate (1080) hazards to fish, wildlife, and invertebrates: A synoptic review. Biological Report 27, Patuxent Environmental Science Centre, U.S. National Biological Service, Laurel, MD. 52p.

Gagnin M.A.H. \& Maravalhas N. 1969. Ocorrência de alcalóides no gênero Palicourea. Anais $20^{\circ}$ Congresso Nacional de Botânica, Goiânia, Goiás, p.91-105.

González B., Suárez-Roca H., Bravo A., Salas-Auvert R. \& Avila D. 2000. Chemical composition and biological activity of extracts from Arrabidaea bilabiata. Pharm. Biol. 38(4):287-290.

Górniak S.L. 1986. Palicourea marcgravii: estudos em animais de laboratório. Dissertação de Mestrado, Faculdade de Medicina Veterinária e Zootecnia, USP, São Paulo. 160p.

Górniak S.L. 1988. Intoxicação por Palicourea marcgravii: uma abordagem experimental. Tese de Doutorado, Faculdade de Medicina Veterinária e Zootecnia, USP, São Paulo. 99p.

Górniak S.L., Souza-Spinosa H., Palermo-Neto J., Ferro V.O. \& Oliveira F. 1986. Chromatographic Isolation of caffeine from Palicourea marcgra vii. Vet. Hum. Toxicol. 28(6):542.

Górniak S.L., Palermo-Neto J. \& Spinosa H.S. 1994. Effects of acetamide on experimentally-induced Palicourea marcgravii (St. Hil.) poisoning in rats. Vet. Hum. Toxicol. 36(2):101-102.

Gregg K., Cooper C. L., Schafer D. J., Sharpe H., Beard C.E., Allen G. \& Xu J. 1994. Detoxification of the plant toxin fluoroacetate by a genetically modified rumen bacterium. Bio/Technology 12:1361-1365.

Gregg K., Hamdorf B., Henderson K., Kopecny J. \& Wong C. 1998. Genetically modified ruminal bacteria protect sheep from fluoroacetate poisoning. Appl. Environ. Microbiol. 64:3496-3498.

Guimarães C.C. 1934. Herva de rato. Vida Médica 2:324-333.

Habermehl G. 1986. Comunicação pessoal. (Apud Górniak 1986)

Helayel M.A., França T.N., Seixas J.N., Nogueira V.A., Caldas S.A. \& Peixoto P.V. 2009. Morte súbita em bovinos causada pela ingestão de Pseudocalymma elegans (Bignoniaceae) no município de Rio Bonito, RJ. Pesq. Vet. Bras. 29(7):498-508.

Helayel M.A., Caldas S.A., Peixoto T.C., França T.N., Tokarnia C.H., Döbereiner J., Nogueira V.A. \& Peixoto P.V. 2011. 0 antagonismo com acetamida em experimentos com ovinos, caprinos e coelhos indica monofluoroacetato como princípio tóxico de Pseudocalymma elegans (Bignoniaceae). Pesq. Vet. Bras. 31(10):867-874. 
Hoehne F.C. 1939. Plantas e Substâncias Vegetais Tóxicas e Medicinais. Graphicars, São Paulo. 356p.

Humphreys D.J. 1988. Veterinary Toxicology. $3^{\text {rd }}$ ed. Bailliere Tindall, London. 356p.

Jabour F.F., Seixas J.N., Tokarnia C.H. \& Brito M.F. 2006. Variação da toxidez de Arrabidaea bilabiata (Bignoniaceae) em coelhos. Pesq. Vet. Bras. 26(3):171-176.

Kemmerling W. 1996. Toxicity of Palicourea marcgravii: Combined effects of fluoroacetate, Nmethyltyramine and 2-methyltetrahydro-beta-carboline. Zeitschrift für Naturforschung 51(1/2):59-64.

Krebs H.C., Kemmerling W. \& Habermehl G. 1994. Qualitative and quantitative determination of fluoroacetic acid in Arrabidaea bilabiata and Palicourea marcgravii by F-NMR spectroscopy. Toxicon 32:909-913.

Mello E.M.M. \& Fernandes J.S. 1940. Contribuição ao Estudo de Plantas Tóxicas Brasileiras. Serviço de Informação Agrícola (SIA), Ministério da Agricultura, Rio de Janeiro. 50p.

Morita H., Ichihara Y., Takeya K., Watanabe K., Itokawa H. \& Motidome M. 1989. A new alkaloid glycoside from the leaves of Palicourea marcgravii. Plantas Medicinais 55(3):288-289.

Nicolella A., Ferreira E.M., Lessa C.A.S., Medeiros M.S.C. \& Lisboa A. 2005. Dados de grupo de agentes, p.69-96. In: Nicolella A., Ferreira E.M., Lessa C.A.S. \& Dallegrave E. (Eds), Toxicovigilância, Toxicologia Clínica. Centro de Informação Toxicológica do Rio Grande do Sul, Porto Alegre.

Nicolella A., Ferreira E.M., Lessa C.A.S. \& Medeiros M.S.C. 2006. Dados de grupo de agentes, p.65-96. In: Nicolella A., Ferreira E.M., Lessa C.A.S. \& Dallegrave E. (Eds), Toxicovigilância, Toxicologia Clínica. Centro de Informação Toxicológica do Rio Grande do Sul, Porto Alegre.

Nicolella A., Ferreira E.M., Lessa C.A.S. \& Medeiros M.S.C. 2007. Dados de grupo de agentes, p.65-96. In: Nicolella A., Ferreira E.M., Lessa C.A.S. \& Dallegrave E. (Eds), Toxicovigilância, Toxicologia Clínica. Centro de Informação Toxicológica do Rio Grande do Sul, Porto Alegre.

Nicolella A., Ferreira E.M., Lessa C.A.S. \& Medeiros M.S.C. 2008/2009. Dados de grupo de agentes, p.67-94. In: Nicolella A., Ferreira E.M., Lessa C.A.S. \& Dallegrave E. (Eds), Toxicovigilância, Toxicologia Clínica. Centro de Informação Toxicológica do Rio Grande do Sul, Porto Alegre.

Nogueira V.A., França T.N., Peixoto T.C., Caldas S.A., Armién A.G. \& Peixoto P. 2010. Intoxicação experimental por monofluoroacetato de sódio em bovinos: aspectos clínicos e patológicos. Pesq. Vet. Bras. 30(7):533-540.

Nogueira V.A., Peixoto T.C., França T.N., Caldas S.A. \& Peixoto P.V. 2011. Intoxicação por monofluoroacetato em animais. Pesq. Vet. Bras. 31(10):823838.

Oliveira M.M. 1963. Chromatographic isolation of monofluoroacetic acid from Palicourea marcgravii, St. Hil. Experientia 19:586.
Oliveira C.M.C., Barbosa J.D., Macedo R.S.C., Brito M.F., Peixoto P.V. \& Tokarnia C.H. 2004. Estudo comparativo da toxidez de Palicourea juruana (Rubiaceae) para búfalos e bovinos. Pesq. Vet. Bras. 24(1):27-30.

Ortis M. 2005. Comissão externa destinada a acompanhar as investigações sobre o envenenamento de animais ocorrido na Fundação Zoológico de São Paulo (envenenamento no Zoológico de São Paulo). Disponível em <www.camara.gov.br/sileg/integras/292702.pdf> Acesso 14 ago. 2009.

Peckolt T. 1868. Herva de rato. Vida Médica 2:324-333. (Apud Guimarães 1934)

Peixoto T.C., Nogueira V.A., Coelho C.D., Veiga C.C.P., Peixoto P.V. \& Brito M.F. 2010. Aspectos clínico-patológicos e laboratoriais da intoxicação experimental por monofluoroacetato de sódio em ovinos. Pesq. Vet. Bras. 30(12):1021-1030.

Peixoto P.V., Nogueira V.A., França T.N., Peixoto T.C., Döbereiner J. \& Tokarnia C.H. 2011a. Relationship between a peculiar form of hydropic-vacuolar degeneration of the distal convoluted tubules, monofluoroacetate poisoning, and plants that cause "sudden death" in Brazil, p.365-372. In: Riet-Correa F., Pfister J., Schild A.L. \& Wierenga T. (Eds), Poisoning by Plants, Mycotoxins, and related Toxins. CABI, London.

Peixoto T.C., Iglesias L., Caldas S.A., Catunda Junior F.E.A., Carvalho M.G., França T.N. \& Peixoto P.V. 2011b. Efeito protetor da acetamida sobre as intoxicações experimentais em ratos por monofluoroacetato de sódio e por algumas plantas brasileiras que causam morte súbita. Pesq. Vet. Bras. 31(11):938-952.

Riet-Correa F. 2011. Comunicação pessoal (Universidade Federal de Campina Grande, Patos, $\mathrm{PB})$.

Soto-Blanco B., Haraguchi M., Silva J.A. \& Górniak S.L. 2004. Intoxicação natural de caprinos e ovinos por Palicourea marcgravii St. Hil. (Rubiaceae). Revta Caatinga, Mossoró, 17(1):52-56.

Tokarnia C.H., Döbereiner J., Canella C.F.C. \& Guimarães D.J. 1969. Intoxicação experimental por Pseudocalymma elegans (Vell.) Kuhlm. em bovinos. Pesq. Agropec. Bras. 4:195-204.

Tokarnia C.H., Peixoto P.V. \& Döbereiner J. 1990. Poisonous plants affecting heart function of cattle in Brazil. Pesq. Vet. Bras. 10(1/2):1-10.

Tokarnia C.H., Döbereiner J. \& Peixoto P.V. 2000. Plantas Tóxicas do Brasil. Editora Helianthus, Rio de Janeiro. 310p.

Tokarnia C.H., Döbereiner J. \& Peixoto P.V. 2002. Poisonous plants affecting livestock in Brazil. Toxicon 40:635-660.

Tokarnia C.H., Barbosa J.D., Oliveira C.M.C., Brito M.F., Oliveira R.B. \& Barbas L.A. 2004. Aspectos epidemiológicos e clínico-patológicos comparados da intoxicação por Arrabidaea bilabiata (Bignoniaceae) em búfalos e bovinos. Pesq. Vet. Bras. 24(2):74-79. 